\title{
Secuestro pulmonar extralobular como causa de hemorragia pulmonar recurrente en un recién nacido
}

\author{
Extralobar pulmonary sequestration as a cause of recurrent pulmonary \\ hemorrhage in a neonate
}

Dra. Arzu Akdag ${ }^{a}$, Dra. Sule Aricib ${ }^{b}$ Dr. Mete Kayac y Dr. Ahmet Turgut ${ }^{d}$

\section{RESUMEN}

El secuestro pulmonar es una malformación pulmonar congénita, quística o sólida, compuesta de tejido pulmonar no funcional sin conexión con elárbol traqueobronquial y que recibe sangre arterial de la circulación sistémica. Existen dos formas de secuestro: intralobular y extralobular. El tratamiento se realiza mediante resección quirúrgica. Se describe el caso de un recién nacido prematuro con secuestro pulmonar extralobular que presentó insuficiencia respiratoria y hemorragia pulmonar recurrente. Luego de la cirugía, el paciente mejoró en forma clínicamente significativa.

Palabras clave: enfermedades pulmonares, hemorragia, secuestro pulmonar, extralobular, recién nacido.

http:/ / dx.doi.org/10.5546/aap.2016.e21

\section{INTRODUCCIÓN}

El secuestro pulmonar (SP) es una lesión congénita que consta de parénquima pulmonar anómalo que recibe su propio suministro de sangre arterial y no tiene conexión con el árbol bronquial normal. Existen principalmente dos tipos de SP: intralobular y extralobular. El secuestro pulmonar intralobular (SPIL) es una masa de parénquima pulmonar contigua al pulmón normal adyacente. En cambio, el secuestro pulmonar extralobular (SPEL) es una masa de parénquima pulmonar con un saco pleural propio que mantiene dicha masa total y anatómicamente separada del tejido pulmonar

a. División de Neonatología.

b. Departamento de Pediatría.

c. Departamento de Cirugía Pediátrica.

d. Departamento de Pediatría.

Hospital de Formación e Investigación Şevket Yılmaz de Bursa, Bursa, Turquía.

Correspondencia:

Dra. Arzu Akdag: arzuakdag@hotmail.com

Financiamiento: Ninguno.

Conflicto de intereses: Ninguno que declarar.

Recibido: 15-7-2015

Aceptado: 31-8-2015 normal adyacente. El SPEL puede presentarse en el mediastino o en el espacio subfrénico., ${ }^{1,2}$

En general, el SPEL es asintomático. El SP sintomático suele ser de tipo intralobular y se presenta como infección, hemoptisis y hemotórax causado por comunicación con las vías aéreas. ${ }^{3}$

En este artículo, se describe el caso de un recién nacido prematuro con SPEL que presentó insuficiencia respiratoria y hemorragia pulmonar recurrente. Luego de la cirugía, el paciente mejoró en forma clínicamente significativa. Hasta donde sabemos, este es el primer informe de un recién nacido con SPEL complicado por hemorragia pulmonar recurrente.

\section{CASO CLÍNICO}

El paciente era un recién nacido varón de 2100 g que nació por cesárea de una madre de 42 años de edad (grávida: 6, para: 6) a las 32 semanas de gestación debido a trabajo de parto prematuro y situación transversa. Fue hospitalizado en la unidad de cuidados intensivos neonatales por dificultad respiratoria grave. No había antecedentes relevantes en la historia clínica materna y familiar.

En el examen físico, el paciente estaba cianótico, tenía reflejos neonatales suprimidos, respiración espontánea deficiente, aleteo nasal y tiraje intercostal. El resto de los exámenes fue normal. Se lo intubó y se lo conectó a un respirador. Se le administró surfactante mediante el tubo endotraqueal ya que la radiografía de tórax reveló un patrón reticulogranular (Figura 1). A los tres días de vida, presentó hemorragia pulmonar. No tenía trombocitopenia, coagulopatías ni conducto arterial persistente, que hubieran contribuido a la hemorragia pulmonar. Inicialmente, se consideró que la hemorragia pulmonar había sido causada por el síndrome de dificultad respiratoria y la prematurez. A pesar de múltiples transfusiones de plasma congelado en fresco e inyecciones de vitamina $K$, se produjeron nuevos episodios de hemorragia pulmonar hasta 
los 21 días de vida. Se le realizó una tomografía computarizada del tórax. Se detectó SPEL en el lóbulo inferior del pulmón izquierdo que recibía sangre de la circulación vascular a través de la aorta torácica descendente (Figura 2). Al paciente se le realizó una cirugía de resección de la lesión mediante una toracotomía posterolateral izquierda estándar. Durante la cirugía, se observó parénquima pulmonar no ventilado en la región basal posterior del tórax izquierdo, que recibía sangre de dos arterias anómalas que surgían de la aorta torácica y drenaban directamente en la vena ácigos (Figura 3). Se realizó una resección completa. La evolución postoperatoria transcurrió sin complicaciones. Luego de la cirugía, se observó mejoría clínicamente significativa. A los tres días de la cirugía, se extubó al paciente, quien recibió el alta a los diez días de la cirugía, tras recuperarse por completo. El paciente continuó un curso asintomático durante los seis meses de seguimiento.

\section{DISCUSIÓN}

La hemorragia pulmonar es una afección potencialmente mortal asociada con muchos factores predisponentes, por ejemplo, menor edad gestacional, presencia de síndrome de dificultad respiratoria, conducto arterial persistente, hipoxia, hipotermia grave y cardiopatía congénita. ${ }^{4} \mathrm{Se}$ ha sugerido que una coagulación deficiente podría simplemente exacerbar o prolongar la hemorragia, no dar lugar a esta. ${ }^{5}$ La evaluación de estos pacientes incluye un hemograma completo,

\section{FIGURA 1. Radiografía de tórax con aspecto reticulogranular}

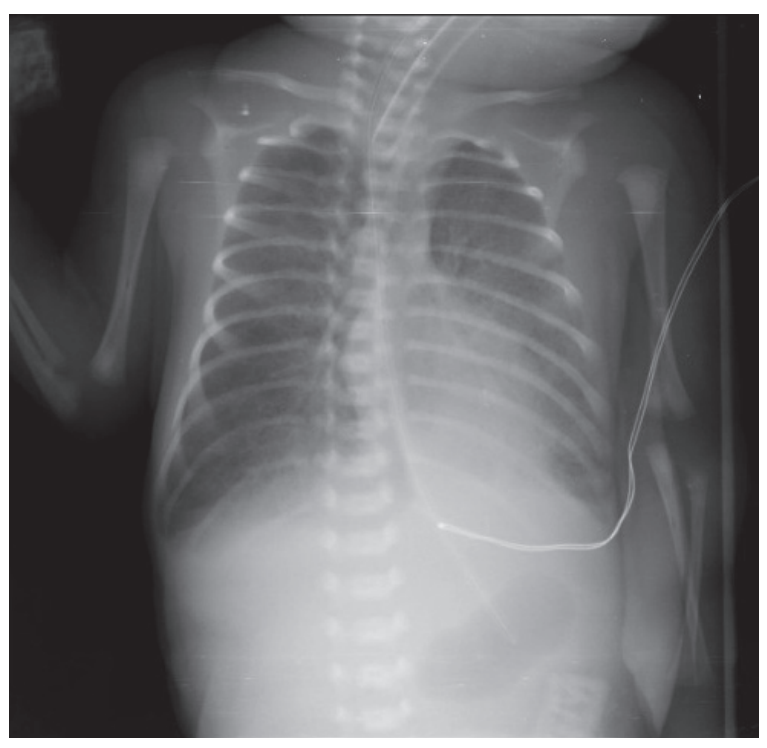

estudios de coagulación, una radiografía de tórax, un ecocardiograma y, de ser necesario, una broncoscopia y una angiografía de las arterias bronquiales. Las opciones de tratamiento luego del deterioro agudo requirieron control de las vías aéreas y estabilización del paciente, con enfoque en la etiología.

El SP es una lesión quística o sólida que consiste en parénquima pulmonar anómalo sin conexión con el árbol bronquial normal con su propio suministro de sangre arterial. Rokitansky y Rektorik ${ }^{2}$ fueron los primeros en describirlo en

FIgURA 2. Tomografía computada de tórax: en el lóbulo inferior izquierdo se observa el secuestro pulmonar extralobular

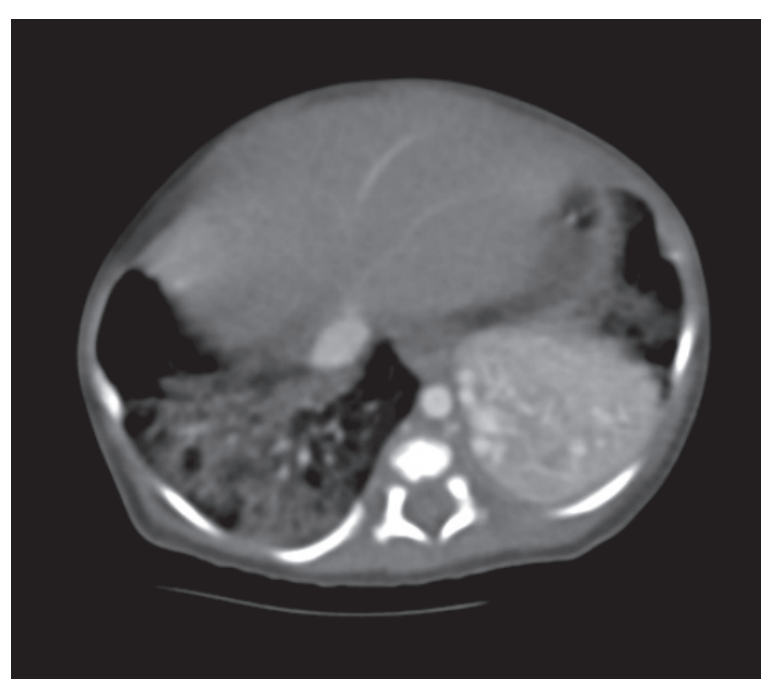

FIGURA 3. Región posterior y basal del lado izquierdo: parénquima pulmonar no ventilado

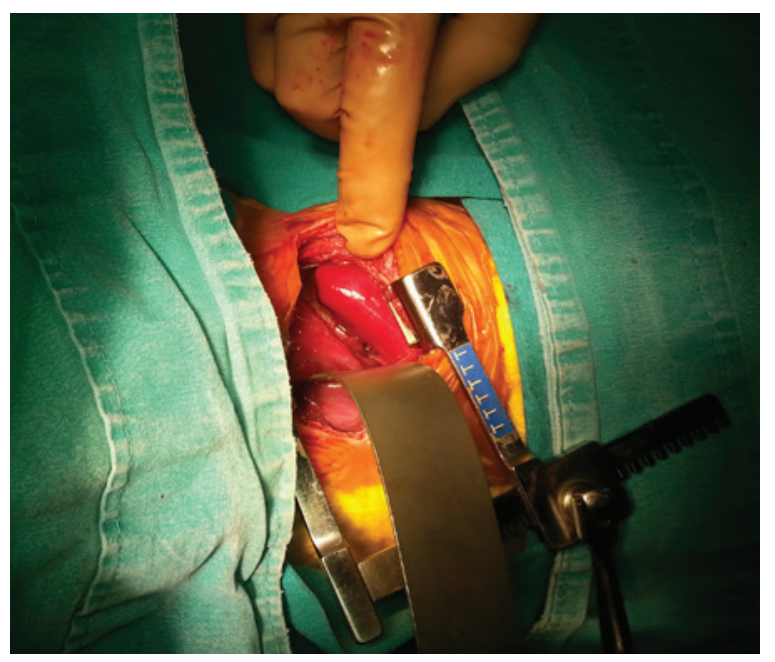


1861. No hay consenso en torno a la etiología del SP, aunque existen dos hipótesis que parecen más probables: formación y migración caudal de un brote pulmonar supernumerario que acompaña el esófago y deficiencia vascular pulmonar primaria, lo que explicaría la persistencia de la circulación sistémica colateral. ${ }^{1,6}$ El SP representa entre el $0,15 \%$ y el $6 \%$ de todas las malformaciones pulmonares congénitas. Estas lesiones se dividen en dos tipos según el saco pleural.

El SPEL es una masa de parénquima pulmonar con un saco pleural propio que mantiene dicha masa total y anatómicamente separada del tejido pulmonar normal adyacente. El SPIL es una masa de parénquima pulmonar contigua al pulmón normal adyacente. El SPIL representa el $75 \%$ de los secuestros, mientras que el SPEL, el 25\%.,

Tanto el SPIL como el SPEL se ubican más frecuentemente en el lóbulo inferior del pulmón izquierdo. El SPEL está más comúnmente asociado con una deformación congénita con cardiopatía congénita, hernia diafragmática, hidropesía, tórax hundido y anomalía vertebral que el SPIL. ${ }^{1,3}$ En nuestro paciente, se detectó SPEL en el lóbulo inferior del pulmón izquierdo sin otras anomalías.

Tanto el SPEL como el SPIL tienen su propio suministro de sangre de circulación vascular proveniente habitualmente de la aorta o de una de sus ramas. En el SPIL, el drenaje venoso se da generalmente en las venas pulmonares, aunque en el SPEL las venas drenan a través del sistema porta o las venas ácigos o hemiácigos. ${ }^{1,7} \mathrm{En}$ nuestro paciente, el suministro de sangre de la circulación vascular provenía de la aorta torácica descendente y las venas drenaban en la vena ácigos.

Las manifestaciones clínicas del SP incluyen fiebre, tos, expectoraciones, dificultad respiratoria, cianosis y hemoptisis. Si bien el SP es mayormente asintomático durante el período neonatal, durante la infancia los pacientes pueden presentar síndrome de dificultad respiratoria grave, insuficiencia cardíaca congestiva, hidrotórax y neumonía. ${ }^{1,3}$ En nuestro paciente, los hallazgos clínicos fueron hemorragia pulmonar e insuficiencia respiratoria poco tiempo después del nacimiento.

Raras veces, el SP puede producir hemorragia pulmonar y hemoptisis. Si bien se desconoce el mecanismo preciso, la hipótesis indica que la hemorragia pulmonar es causada por la degeneración vascular provocada por la compresión pulmonar del SP. ${ }^{8}$ En este paciente con SPEL, la lesión había comprimido la vasculatura pulmonar, lo que produjo la hemorragia pulmonar.

En el SP, las modalidades de tratamiento dependen de si el paciente tiene dificultad respiratoria, infecciones recurrentes o no presenta síntomas. El tratamiento de los pacientes asintomáticos o de los que tienen SP detectado casualmente es controversial. En el SPIL suele recomendarse la resección para prevenir complicaciones, aunque aún no se ha establecido el momento óptimo para realizar la cirugía. Por otro lado, en los pacientes sintomáticos, se recomienda la resección de la lesión. En general, el SPIL se trata mediante lobulectomía o resección segmentaria, mientras que el SPEL puede extirparse mediante resección simple, ya que la lesión tiene su propia pleura y suministro sanguíneo.

Sin embargo, el suministro de sangre arterial del SPEL podría provenir de la aorta subdiafragmática, por lo que es fundamental identificar los vasos. De manera alternativa, puede ofrecerse una toracoscopia o radiología intervencionista como tratamiento para los pacientes con SP. El avance en las técnicas de cirugía fetal ha permitido que la intervención prenatal sea una opción valiosa en casos selectos de SP. ${ }^{8-10}$ En nuestro paciente, se extirpó la lesión satisfactoriamente mediante una toracotomía abierta.

A modo de conclusión, el SP puede producir hemorragia pulmonar recurrente durante el período neonatal. Debe considerarse el SP cuando no es posible diferenciar el origen de una hemorragia pulmonar mediante un examen no invasivo. Pueden realizarse una aortografía, una angiografía Doppler y una resonancia magnética en los pacientes en los que se sospecha esta entidad. La tomografía computarizada también podría aportar información valiosa; en estos casos, la resección quirúrgica de la lesión podría salvar vidas.

\section{REFERENCIAS}

1. Pires CR, Czapkowski A, Araujo Júnior E, Zanforlin Filho SM. Diagnosis of Intra-abdominal extralobar pulmonary sequestration by means of ultrasound in a neonate. Case Rep Pediatr 2013;2013:623102.

2. Tetsuka K, Endo S, Kanai Y, Yamamoto S. Extralobar pulmonary sequestration presenting as hemothorax. Interact Cardiovasc Thorac Surg 2009;9(3):547-8.

3. Ou J, Lei X, Fu Z, Huang Y, et al. Pulmonary sequestration in children: a clinical analysis of 48 cases. Int J Clin Exp Med 2014;7(5):1355-65.

4. Raju TN, Langenberg P. Pulmonary hemorrhage and 
exogenous surfactant therapy: a metaanalysis. J Pediatr 1993;123(4):603-10.

5. Cole VA, Normand IC, Reynolds EO, Rivers RP. Pathogenesis of hemorrhagic pulmonary edema and massive pulmonary hemorrhage in the newborn. Pediatrics 1973;51(2):175-87.

6. Morin L, Crombleholme TM, D'Alton ME. Prenatal diagnosis and management of fetal thoracic lesions. Semin Perinatol 1994;18(3):228-53.

7. Cakir U, Kahvecioglu D, Alan S, Yildiz D, et al. Extralobar pulmonary sequestration requiring intrauterine thoracentesis. APSP J Case Rep 2015;6(1):3.

8. Reynolds M. Congenital lesions of thelung. En:Shields TW, LoCicero J, Ponn RP, eds. General thoracic surgery. 5th ed. Philedelphia: Lippincott Williams \& Wilkins; 2000:937-52.

9. Laberge JM, Puligandla P, Flageole H. Asymptomatic congenital lung malformations. Semin Pediatr Surg 2005;14(1):16-33.

10. Lee BS, Kim JT, Kim EA, Kim KS, et al. Neonatal pulmonary sequestration: clinical experience with transumbilical arterial embolization. Pediatr Pulmonol 2008;43(4):404-13. 